\title{
Dynamic Self-Assembly of "Living” Polymeric Chains
}

\author{
Binghui Deng and Yunfeng Shi* \\ Department of Materials Science and Engineering \\ Rensselaer Polytechnic Institute, Troy, NY 12180, United States
}

Keyword: molecular dynamics, polymeric chains, dynamic self-assembly.

\begin{abstract}
We report a dynamic self-assembly system of "living" polymeric chains sustained by chemistry using reactive molecular dynamics simulations. The linear polymeric chains consist of self-assembled nanoparticles connected by metastable linker molecules. As such, the polymeric chains, once assembled, undergo spontaneous dissociation driven by thermodynamics. However, with a continuous supply of linker molecules and the stored chemical energy therein, the polymeric chains can survive and maintain a steady state averaged chain length. These dynamically self-assembled polymeric chains are analogous to biological systems that both are thermodynamically metastable, yet dynamically stable upon continuous influx of matter and energy.
\end{abstract}

Self-assembly, nature's versatile and creative way to produce its creations, generally involves processes of spontaneous formation of ordered/organized structures from discrete building blocks interacting directly with each other or indirectly via the surrounding environment [1-3]. The ubiquitous demonstrations of self-assembly can be seen in nature at all length scales, from DNA strands [4] to Saturn rings [5]. Inspired by nature, a wide range of artificial selfassembly systems have been reported over the last few decades, such as 2D and 3D structures bonded by capillary forces [6,7], process-directed self-assembled copolymers [8], and ferrofluid droplets [9] driven by magnetic field. Despite the advancements exemplified as above, self-assembly is still far from being completely understood, nor can it be designed as versatile and sophisticated as it is in nature. The need to deepen our understanding of self-assembly becomes increasingly urgent on the heels of some significant progress made in fabricating nanoscale building blocks with everincreasing control over the size, shape and composition in recent years [10-13].

Most of the existing work and current understanding of self-assembly are limited to static systems dictated by thermodynamics $[1,3,14,15]$, i.e., static self-assembly (SSA) systems. Different from SSA, dynamic self-assembly (DySA) systems are formed spontaneously in dissipative systems that are (for instance, sustained chemical/mechanical stimuli) away from thermodynamic equilibrium [3]. Despite some insightful early researches [16-20], little understanding has been obtained regarding the general design principles of DySA systems. Instead, DySA systems appear to be strongly system-specific. To this end, chemically sustained DySA systems have received little attention, yet are ubiquitous in nature.

Computer simulations have been widely used to study self-assembly, in which nanoscale building blocks with prescribed structure, composition and interaction can be constructed and the precise assembly process for each atom can be known at every instant. Up to date even though there have been a considerable number of simulation studies on self-assembly of various systems, including nanoparticle [21], lipid bilayer [22], polymer [23,24], DNA [25], and micelles [26], there are very few simulation reports on DySA systems.

Here we report a molecular dynamic (MD) simulation on DySA system of "living" polymeric chains sustained by chemistry. The system is in two dimensions for simplicity. It consists of three types of particles: A, B and C, representing linkers (ligands) and anchors (nanoparticles). The force field used here is a modified Lennard-Jones $(\mathrm{mLJ})$ potential $[27,28]$, which differs from the conventional smoothed $\phi_{L J}^{\text {smooth }}(r)$ by the inclusion of a repulsive bump term $b(r)$ outside of the potential well. The pair-wise interaction of ${ }_{m L J}(r)$ can be written as, 


$$
\begin{aligned}
& \phi_{m L J}(r)= \begin{cases}\phi_{L J}^{\text {smooth }}(r), & r<r_{\alpha \beta}^{b} \\
\phi_{L J}^{\text {smooth }}(r)+b(r), & r \geq r_{\alpha \beta}^{b} \\
0, & r \geq r_{\alpha \beta}^{c}\end{cases} \\
& \phi_{L J}^{\text {smooth }}(r)=\phi_{L J}(r)-\phi_{L J}\left(r_{\alpha \beta}^{c}\right)-\left(r-r_{\alpha \beta}^{c}\right) \phi_{L J}^{\prime}\left(r_{\alpha \beta}^{c}\right) \\
& \phi_{L J}(r)=4 \varepsilon_{\alpha \beta}\left(\frac{\sigma_{\alpha \beta}^{12}}{r^{12}}-\frac{\sigma_{\alpha \beta}^{6}}{r^{6}}\right) \\
& b(r)=\varepsilon_{\alpha \beta}^{b} \varepsilon_{A B} \cdot \sin ^{2}\left(\pi \frac{r_{\alpha \beta}^{c}-r}{r_{\alpha \beta}^{c}-r_{\alpha \beta}^{b}}\right)
\end{aligned}
$$

where $r_{\alpha \beta}^{c}$ is the pair dependent cutoff ( $\alpha, \beta$ denotes species of $\mathrm{A}, \mathrm{B}$ or $\mathrm{C})$. The smoothed LJ function $\phi_{L J}^{\text {smooth }}(r)$ ensures that both the potential energy and its first derivative are continuous at $r_{\alpha \beta}^{c}$. The bump term $b(r)$ is only applied in the range of $r_{\alpha \beta}^{b}$ and $r_{\alpha \beta}^{c} . \varepsilon_{\alpha \beta}^{b}$ is the height of the repulsive bump. Note that the bump term itself and its first derivative are zero at both $r_{\alpha \beta}^{c}$ and $r_{\alpha \beta}^{b}$. Therefore, the overall $\mathrm{mLJ}$ potential is still smooth after the inclusion of $b(r)$ at $r_{\alpha \beta}^{c}$ and $r_{\alpha \beta}^{b} \cdot \varepsilon_{A B}$ and $\sigma_{A B}$ provide the energy and length scales for the pair interaction, respectively. The mass of all three types of particles is $m_{0}$. Accordingly, the time scale of the simulation system $t_{0}$ equals to $\sigma_{A B} \sqrt{m_{0} / \varepsilon_{A B}}$.

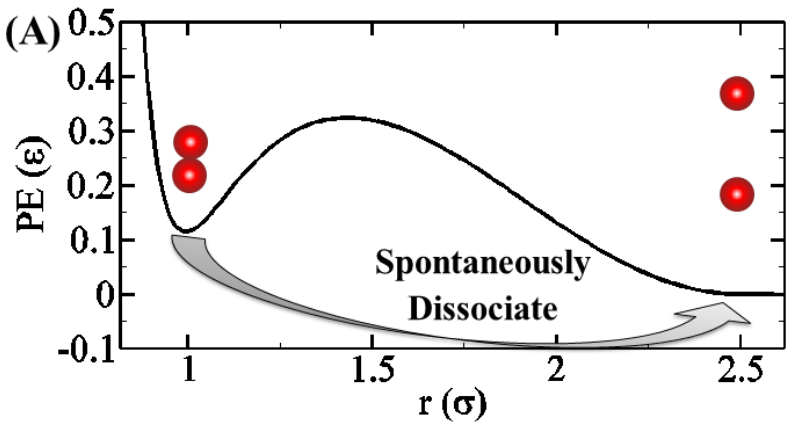

(B)

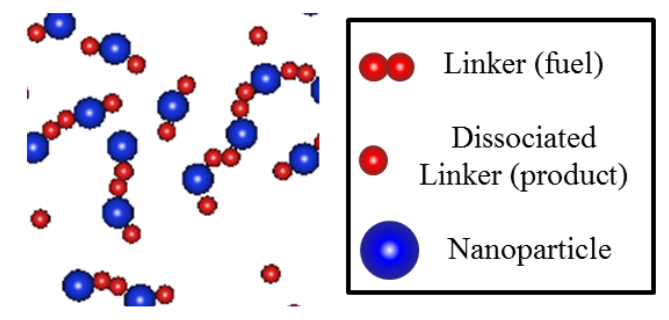

Figure 1. (A) Potential profile indicating spontaneous dissociation of the linker molecules (A-A pair interaction). (B) A snapshot of the simulation system with linkers (B-A-A-B molecules as the pairs of red particles) and nanoparticles (C particles, in blue). As B particles can be considered as patches to A particles, they are not shown for the sake of clarity.
The parameters of the force field to represent the linkeranchor (ligand-nanoparticle) system are listed in Table 1. The linker molecules are linear molecules of B-A-A-B. The bump term is exclusively applied to A-A interaction from zero up to the cutoff with a large value aiming at lifting the original potential well above zero. A-A bond is therefore metastable (Figure 1A) and tends to dissociate spontaneously driven by thermodynamics. B particles bond strongly to A particles, yet repel other B particles (purely repulsive). Thus, they serve as patches of A particles to ensure that any given single A particle could at most bond one more A particle. C particles represent generic nanoparticles, which only bond with B particles. Similar to the B-B interaction, both A-C and $\mathrm{C}-\mathrm{C}$ interaction are purely repulsive, such that any given single $C$ particle can bond at most two linker molecules of B-A-A-B. A polymeric chain consisting of the linkers and anchors is therefore essentially built up by alternating the linear molecules and the nanoparticles: -B-A-A-B-C-B-A-AB-C-B-A-A-B-, as shown in Figure 1(B). Note that B particles are essentially patches of A particles, they are not displayed in Figure 1 for the sake of clarity.

Table 1. Species-dependent parameters of the $\mathrm{mLJ}$ force field for various pairs of particles.

\begin{tabular}{llllll}
\hline & $\varepsilon_{\alpha \beta} / \varepsilon_{A B}$ & $/{ }_{A B}$ & $\varepsilon_{\alpha \beta}^{b} / \varepsilon_{A B}$ & $r^{b} /{ }_{A B}$ & $r_{\alpha \beta}^{c} / \sigma_{A B}$ \\
\hline A-A & 0.5 & 0.900 & 1.6 & 0.0 & 2.5 \\
B-B & 0.5 & 1.782 & 0.0 & 1.3 & 2.0 \\
A-B & 2.0 & 0.445 & 0.0 & 0.8 & 1.3 \\
A-C & 1.0 & 1.336 & 0.0 & 1.4 & 1.5 \\
B-C & 0.3 & 0.900 & 0.0 & 1.3 & 2.5 \\
C-C & 0.5 & 2.227 & 0.0 & 1.4 & 2.5 \\
\hline
\end{tabular}

The simulation box size is set up as $200_{A B} \quad 200_{A B}$ with periodic boundary conditions. It contains 1600 nanoparticles and 1600 linker molecules in total. Over the course of the simulation, the equations of motions are numerically integrated using the velocity Verlet algorithm [29] with a time step of $0.01 t_{0}$. As the linker molecules dissociate (B-A-A-B $\rightarrow 2$ B-A), the concentration of the linker molecules consequently decreases over time. In order to study the DySA in the steady state, a product-to-reactant refueling operation (2 B-A $\rightarrow$ B-A-A-B) is invoked during the simulation. This refueling operation is essentially equivalent to constantly introducing chemical fuels (B-A-A$\mathrm{B}$ linker molecules) and removing products (B-A molecules). To implement this, two isolated B-A molecules are first randomly selected, and then an attempt will be made to reposition the first B-A molecule to the adjacency of the 
other one to form a B-A-A-B molecule in a straight line. If the attempted displacement does not lead to any particle overlap (no two atoms are within $1.5 \sigma_{A B}$ ), this displacement attempt will be accepted, and vice versa. A similar refueling operation has been implemented in our previous studies [3032]. Note that the product-to-reactant refueling operation does not alter the velocities of the involved particles, and the attempt frequency defines a maximum allowed refueling rate, which is used to control the strength of the chemical dissipation of the simulation system.

Figure 2 shows the drastic difference between simulation systems without the exchange of chemicals thus evolving towards equilibrium (A), and with the exchange of chemicals thus evolving towards a non-equilibrium steady state (B). For both cases, the linker molecules can dissociate and bind to nanoparticles, but the dissociation rate is much slower than the binding rate. This is due to the presence of an activation barrier for the dissociation reaction as shown in Figure 1. For the first case (Figure 2A) towards equilibrium in the closed isothermal system, the system first forms polymeric chains due to the system initial configuration, and then the linkers begin to slowly dissociate, resulting in very few polymeric chains left as the simulation proceeds. In other words, the polymeric chains tend to die out under equilibrium conditions. In strong contrast, for the second case (Figure 2B) towards non-equilibrium steady state with the exchange of chemicals (chemical dissipation), polymeric chains not only self-assemble but also dynamically sustain in this open isothermal system.
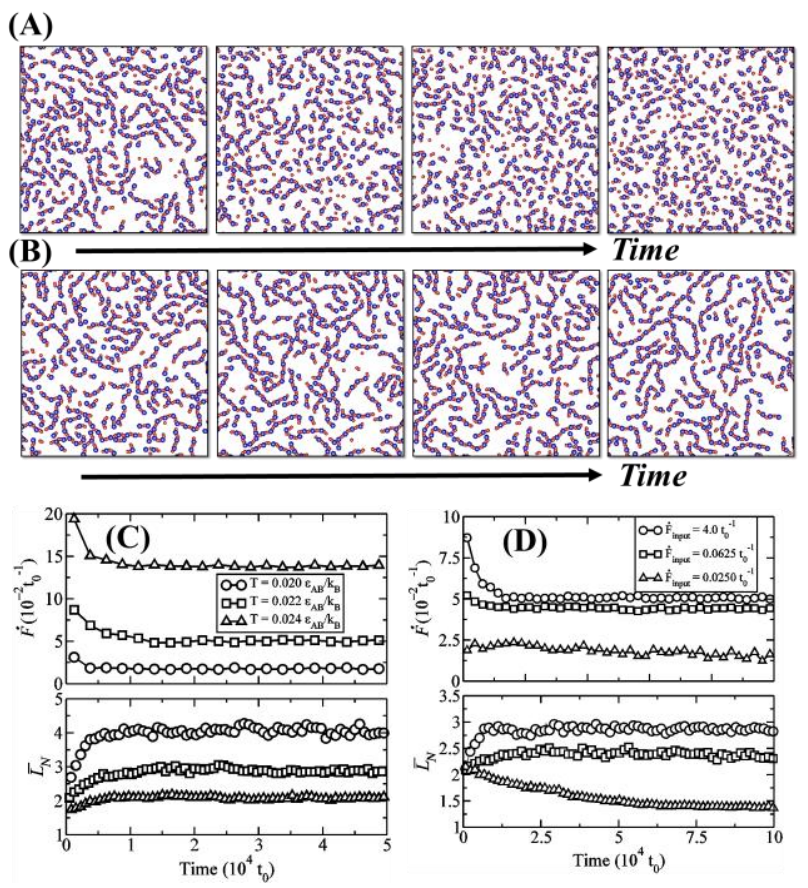

Figure 2. (A) System evolution of dynamic self-assembly of polymeric chains without the refueling process at $\mathrm{T}=0.022$ $\varepsilon_{A B} / k_{B}$. Driven by thermodynamics, polymeric chains first form, then later on die out. (B) In contrast, polymeric chains are well sustained with constant energy influx via refueling

operation. (C) The actual refueling rate $(\dot{F})$ and averaged chain length $\left(\bar{L}_{N}\right)$ evolution for systems with the same maximum

allowed refueling rate $\left(4.0 t_{0}{ }^{1}\right)$ at different temperatures. (D)

The $\dot{F}$ and $\bar{L}_{N}$ evolution for systems with the various maximum allowed refueling rate at the temperature of 0.022 ${ }_{A B} / k_{B}$.

Interestingly, this DySA system can be tuned by varying the thermal and chemical environments. Figure 2(C) shows a set of simulations that have identical maximum allowed refueling rate at different temperatures. The system dynamically self-assembles into different steady states (different $\bar{L}_{N}$ ), depending on the temperature. The dissociation rate of the linker molecules increases with the increasing temperature, thus longer chains can survive at low temperatures. In return, since longer chains contain more fuels (B-A-A-B molecules) and fewer products (B-A molecules), and the refueling operation is only feasible when isolated product molecules are available, the actual refueling rate is lower than those shorter chain systems. It is also worthwhile to point out that the maximum allowed refueling rate defines the upper limit to the actual refueling rate that quantifies the actual dissipative chemical force. Similarly, Figure 2(D) shows a set of simulations with different maximum allowed refueling rates at the same temperature. The actual refueling rate increases with the maximum allowed refueling rate, which leads to more fuels in the system and therefore longer chains. All the systems in Figure $2(\mathrm{C}, \mathrm{D})$ reach steady state conditions with constant averaged chain length, and actual refueling rate.

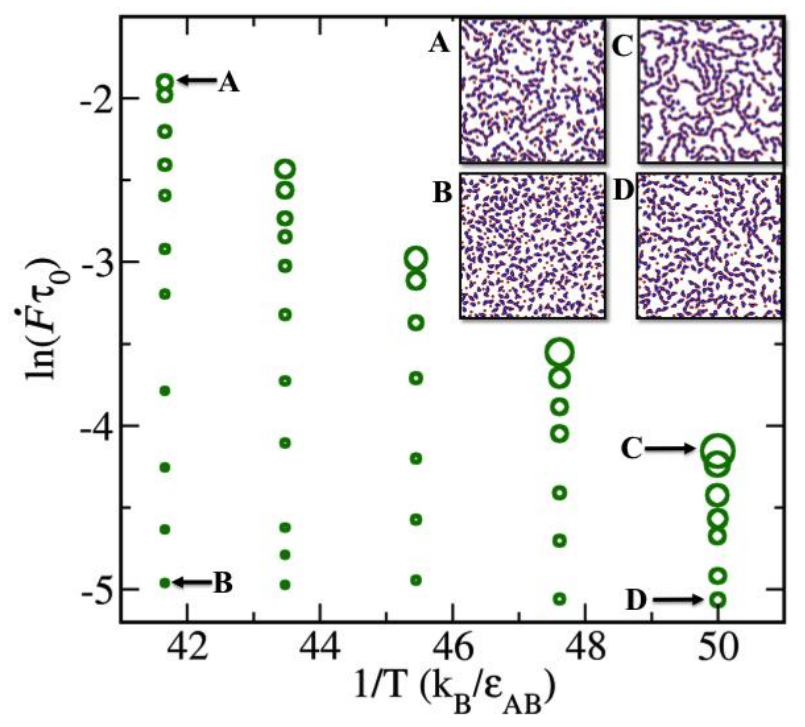


Figure 3. The dynamically self-assembled averaged chain length as a function of temperature and actual refueling rate. Each circle represents a non-equilibrium steady state, with the circle size proportional to the averaged chain length. Four inset graphs show the system configurations with different temperature/maximum refueling rate combinations.

Based on the results shown in Figure 2(C, D), we further systematically explored how thermal and chemical environments affect the DySA behavior. Individual simulations with specific temperature and maximum allowed refueling rate were carried out in which the steady states of self-assembly were reached. Due to the limited computing resources, simulations were run at relatively high temperatures for the ease of achieving steady states. Figure 3 shows the steady state averaged chain length map in the temperature and the actual refueling rate dimensions. It can be clearly seen that high refueling rate or low temperature leads to the formation of longer chains, and vice versa. For systems in the steady state, since the dissociation of the linkers has to be exactly compensated by the refueling of reactants, systems with higher refueling rates at the same temperature have to contain more linkers (thus longer chains) so that the dissociation rate can match the refueling rate. Similarly, at lower temperatures (thus lower dissociation rates) but with the same refueling rate, systems must also contain more linkers (thus longer chains) to achieve steady states.

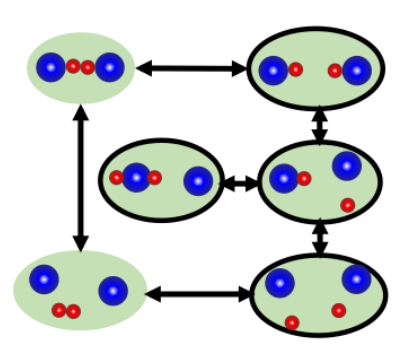

(A)

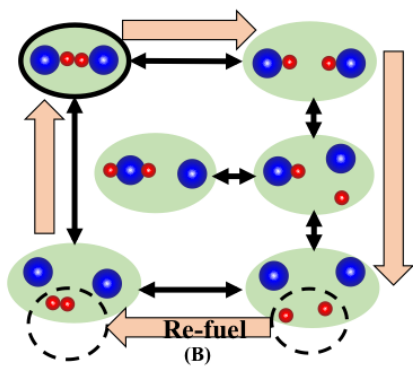

Figure 4. The reaction pathway diagram for the equilibrium system (A) and the non-equilibrium steady state system (B). The representation of chemical species follows the convention in Figure 1. Black double-arrows denote equal energy and matter fluxes in both directions that are required in detailed balance. The one-way arrow denotes the flux from the refueling process and the additional flux it causes. High probability states are marked by thick oval outlines.

To better understand the dynamic self-assembly of the polymeric chains, Figure 4 shows both the reaction pathway diagram of the chemical system at equilibrium (Figure 4A) and at non-equilibrium steady state with the chemical refueling process (Figure 4B). Since the dissociation of linker molecules is both enthalpically and entropically favorable, the dissociation rate of the linker molecules is much higher than the combination rate from the product molecules back to linker molecules. Therefore, under equilibrium, the dominant configurations are dissociated molecules (all four black circles in Figure 4A) instead of long polymeric chains. In other words, the system contains few linkers with high dissociation rate, which is balanced by many products with low combination rate, to achieve equal flux along both directions to satisfy detailed balance. This is why long polymer chains cannot survive under equilibrium. The chemical dissipative force from the refueling process acts on the reaction pathway diagram by imposing an additional flux (the bottom arrow in Figure 4B). This extra flux from isolated product molecules to linker molecules has a ripple effect on additional fluxes among other reactive states. For instance, the extra population of isolated linker molecules leads to additional polymerization flux and higher population of polymeric chains. Note that the fluxes caused by the chemical refueling process are not balanced by its reverse, and result in a loop or vortex in the phase space, such that the populations of each chemical states do not vary with time in steady state.

It should be emphasized that "living" polymeric chains are not static self-assembly with non-equilibrium agitation. The difference is that, the non-equilibrium agitation in static self-assembly facilitates the system to achieve an equilibrium structure, while the non-equilibrium agitation in dynamic self-assembly forces the system to a steady state structure, out of equilibrium. In our simulation system, the equilibrium structure is an almost complete disintegration of the polymeric chains. It is the chemical agitation that drives the system to the self-assembled chain structures, away from equilibrium, while producing entropy (exothermic heat release, and the increase in configurational entropy from spontaneous dissociation of linker molecules). From the perspective of the nanoparticles, they do not interact with each other unless there is a connecting linker molecule inbetween. Therefore, the linker molecules serve somewhat as the dynamic interaction between the nanoparticles, which is essential for dynamic self-assembly. Such dynamic "chemical" interaction is analogous to a variety of other dynamic forces, such as vortex-vortex hydrodynamic interaction, flow-induced viscous interaction, and induced magnetic dipole-dipole interaction [3].

In summary we have designed a dynamic self-assembly system of "living" polymeric chains. The chains can only survive (maintaining the connectivity between monomers) by continuously refueling the linker molecules. The polymeric chains will gradually die out due to the spontaneous dissociation of those linkers driven by thermodynamics. These dynamically self-assembled polymeric chains are analogous to biological systems that 
both are thermodynamically metastable, yet dynamically stable upon continuous influx of matter and energy. On a last note, a self-assembled nanoparticle system with programmable DNA as degradable linkers is a promising experimental analog of the model system described here [33].

\section{Corresponding Author}

*E-mail: shiy2@rpi.edu

\section{ACKNOWLEDGMENTS}

We gratefully acknowledge the support from the National Science Foundation (NSF) under grant DMR-1207439. We thank stimulating discussions with Pawel Keblinski, Rahmi Ozisik, Edmund Palermo, and Linda Schadler from Rensselaer. The MD simulations were carried out in the Center for Computational Innovations (CCI) at Rensselaer.

\section{REFERENCES}

[1] G.M. Whitesides, B. Grzybowski, Self-assembly at all scales, Science. 295 (2002) 2418-2421. doi:10.1126/science.1070821.

[2] B.A. Grzybowski, H.A. Stone, G.M. Whitesides, Dynamic selfassembly of magnetized, millimetre-sized objects rotating at a liquid-air interface, Nature. 405 (2000) 1033-1036.

[3] M. Fialkowski, K.J.M. Bishop, R. Klajn, S.K. Smoukov, C.J. Campbell, B.A. Grzybowski, Principles and implementations of dissipative (dynamic) self-assembly, J. Phys. Chem. B. 110 (2006) 2482-2496. doi:10.1021/jp054153q.

[4] S.L. Lai, D. Hartono, K.-L. Yang, Self-assembly of cholesterol DNA at liquid crystal/aqueous interface and its application for DNA detection, Appl. Phys. Lett. 95 (2009) 153702. doi:10.1063/1.3247895.

[5] R. Greenberg, A. Brahic, Planetary Rings, Arizona University Press, Tucson, AZ, 1984.

[6] T.D. Clark, J. Tien, D.C. Duffy, K.E. Paul, G.M. Whitesides, Self-assembly of 10-mu m-sized objects into ordered threedimensional arrays, J. Am. Chem. Soc. 123 (2001) 7677 7682. doi:10.1021/ja0106341.

[7] S.R.J. Oliver, T.D. Clark, N. Bowden, G.M. Whitesides, Threedimensional self-assembly of complex, millimeter-scale structures through capillary bonding, J. Am. Chem. Soc. 123 (2001) 8119-8120. doi:10.1021/ja010845q.

[8] M. Mueller, D.-W. Sun, Process-directed self-assembly of block copolymers: a computer simulation study, J. Phys.-Condens. Matter. 27 (2015) 194101. doi:10.1088/0953$8984 / 27 / 19 / 194101$.
[9] J.V.I. Timonen, M. Latikka, L. Leibler, R.H.A. Ras, O. Ikkala, Switchable Static and Dynamic Self-Assembly of Magnetic Droplets on Superhydrophobic Surfaces, Science. 341 (2013) 253-257. doi:10.1126/science.1233775.

[10] C.R. Barry, N.Z. Lwin, W. Zheng, H.O. Jacobs, Printing nanoparticle building blocks from the gas phase using nanoxerography, Appl. Phys. Lett. 83 (2003) 5527-5529. doi:10.1063/1.1637143.

[11] V. Pachauri, K. Kern, K. Balasubramanian, Template-free self-assembly of hierarchical $\mathrm{ZnO}$ structures from nanoscale building blocks, Chem. Phys. Lett. 498 (2010) 317-322. doi:10.1016/j.cplett.2010.08.078.

[12] J. Tumpane, P. Sandin, R. Kumar, V.E.C. Powers, E.P. Lundberg, N. Gale, P. Baglioni, J.-M. Lehn, B. Albinsson, P. Lincoln, L.M. Wilhelmsson, T. Brown, B. Norden, Addressable high-information-density DNA nanostructures, Chem. Phys. Lett. 440 (2007) 125-129. doi:10.1016/j.cplett.2007.04.018.

[13] J.G. Wen, J.Y. Lao, D.Z. Wang, T.M. Kyaw, Y.L. Foo, Z.F. Ren, Self-assembly of semiconducting oxide nanowires, nanorods, and nanoribbons, Chem. Phys. Lett. 372 (2003) 717-722. doi:10.1016/S0009-2614(03)00485-8.

[14] C.J. Brinker, Y. Lu, A. Sellinger, H. Fan, Evaporation-Induced Self-Assembly: Nanostructures Made Easy, Adv. Mater. 11 (1999) 579-585. doi:10.1002/(SICI)15214095(199905)11:7<579::AID-ADMA579>3.0.CO;2-R

[15] H. Hess, Self-assembly driven by molecular motors, Soft Matter. 2 (2006) 669-677. doi:10.1039/B518281F.

[16] B.A. Grzybowski, C.J. Campbell, Complexity and dynamic self-assembly, Chem. Eng. Sci. 59 (2004) 1667-1676.

[17] N.H.P. Nguyen, E. Jankowski, S.C. Glotzer, Thermal and athermal three-dimensional swarms of self-propelled particles, Phys. Rev. E. 86 (2012) 011136. doi:10.1103/PhysRevE.86.011136.

[18] S. Soh, M. Branicki, B.A. Grzybowski, Swarming in Shallow Waters, J. Phys. Chem. Lett. 2 (2011) 770-774. doi:10.1021/jz200180z.

[19] A. Coskun, M. Banaszak, R.D. Astumian, J.F. Stoddart, B.A. Grzybowski, Great expectations: can artificial molecular machines deliver on their promise?, Chem. Soc. Rev. 41 (2011) 19-30. doi:10.1039/C1CS15262A.

[20] D.J. Evans, A. Baranyai, Possible variational principle for steady states far from equilibrium, Phys. Rev. Lett. 67 (1991) 2597-2600. doi:10.1103/PhysRevLett.67.2597.

[21] L.-T. Yan, X.-M. Xie, Computational modeling and simulation of nanoparticle self-assembly in polymeric systems: Structures, properties and external field effects, Prog. Polym. Sci. 38 (2013) 369-405. doi:10.1016/j.progpolymsci.2012.05.001. 
[22] A.A. Skjevik, B.D. Madej, C.J. Dickson, C. Lin, K. Teigen, R.C. Walker, I.R. Gould, Simulation of lipid bilayer selfassembly using all-atom lipid force fields, Phys. Chem. Chem. Phys. 18 (2016) 10573-10584.

doi:10.1039/c5cp07379k.

[23] W. Shinoda, R. DeVane, M.L. Klein, Computer simulation studies of self-assembling macromolecules, Curr. Opin. Struct. Biol. 22 (2012) 175-186. doi:10.1016/j.sbi.2012.01.011.

[24] Y.A. Kriksin, I.V. Neratova, P.G. Khalatur, A.R. Khokhlov, Pattern multiplication by template-guided self-assembly of cylinder-forming copolymers: Field-theoretic and particlebased simulations, Chem. Phys. Lett. 492 (2010) 103-108. doi:10.1016/j.cplett.2010.04.028.

[25] J.P.K. Doye, T.E. Ouldridge, A.A. Louis, F. Romano, P. Sulc, C. Matek, B.E.K. Snodin, L. Rovigatti, J.S. Schreck, R.M. Harrison, W.P.J. Smith, Coarse-graining DNA for simulations of DNA nanotechnology, Phys. Chem. Chem. Phys. 15 (2013) 20395-20414. doi:10.1039/c3cp53545b.

[26] Y. Wang, B. Li, Y. Zhou, Z. Lu, D. Yan, Dissipative particle dynamics simulation study on the mechanisms of selfassembly of large multimolecular micelles from amphiphilic dendritic multiarm copolymers, Soft Matter. 9 (2013) 32933304. doi:10.1039/c3sm27396b.

[27] Y. Shi, J. Luo, F. Yuan, L. Huang, Intrinsic ductility of glassy solids, J. Appl. Phys. 115 (2014) 043528. doi:10.1063/1.4862959.

[28] B. Deng, Y. Shi, A reactive coarse-grained model for polydisperse polymers, Polymer. 98 (2016) 88-99. doi:10.1016/j.polymer.2016.06.018.

[29] K. Binder, J. Horbach, W. Kob, W. Paul, F. Varnik, Molecular dynamics simulations, J. Phys. Condens. Matter. 16 (2004) S429.

[30] Y. Chen, Y. Shi, Characterizing the Autonomous Motions of Linear Catalytic Nanomotors Using Molecular Dynamics Simulations, J. Phys. Chem. C. 115 (2011) 19588-19597. doi:10.1021/jp206087p.

[31] Y. Chen, Y. Shi, Dynamic self assembly of confined active nanoparticles, Chem. Phys. Lett. 557 (2013) 76-79. doi:10.1016/j.cplett.2012.11.073.

[32] Q. Xiao, Y. Chen, T. Bereau, Y. Shi, An in-silico walker, Chem. Phys. Lett. 659 (2016) 6-9. doi:10.1016/j.cplett.2016.06.019.

[33] W.B. Rogers, W.M. Shih, V.N. Manoharan, Using DNA to program the self-assembly of colloidal nanoparticles and microparticles, Nat. Rev. Mater. 1 (2016) 16008. doi:10.1038/natrevmats.2016.8. 


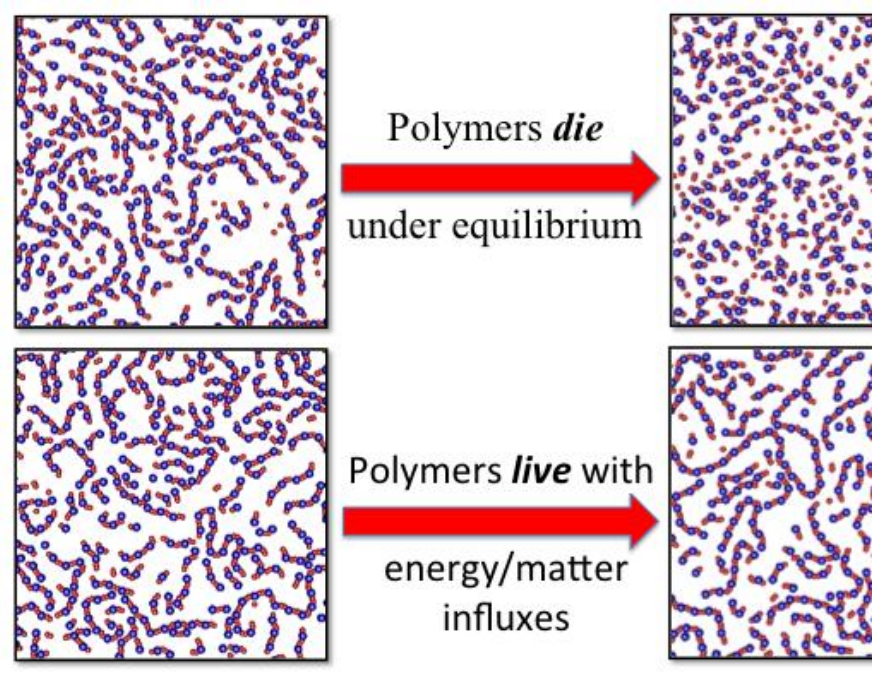

